\title{
NONLINEAR DYNAMIC ANALYSIS OF A RECTANGULAR PLATE SUBJECTED TO ACCELERATED/DECELERATED MOVING LOAD
}

\author{
Ahmad Mamandi \\ Department of Mechanical Engineering, Parand Branch, Islamic Azad University, Tehran, Iran \\ e-mail:am_2001h@yahoo.com \\ Ruhollah Mohsenzadeh \\ Department of Mechanical and Aerospace Engineering, Science and Research Branch, Islamic Azad University, Tehran, Iran \\ Mohammad H. Kargarnovin \\ Department of Mechanical Engineering, Sharif University of Technology, Tehran, Iran
}

\begin{abstract}
In this paper, nonlinear dynamical behavior of a rectangular plate traveled by a moving mass as well as an equivalent concentrated force with non-constant velocity is studied. The nonlinear governing coupled partial differential equations (PDEs) of motion are derived by energy method using Hamilton's principle based on the large deflection theory in conjuncture with the von-Karman strain-displacement relations. Then Galerkin's method is used to transform the equations of motion into a set of three coupled nonlinear ordinary differential equations (ODEs) which then is solved in a semi-analytical way to get the dynamical response of the plate. Also, by using the Finite Element Method (FEM) with ANSYS software, the obtained results in nonlinear form are verified by FEM results. Then, a parametric study is conducted by changing the size of moving mass/force and the velocity of the traveling mass/force with a constant acceleration/deceleration, and the outcome nonlinear results are compared to the results from linear solution.
\end{abstract}

Keywords: nonlinear dynamic, plate, moving load, accelerated/decelerated motion

\section{Introduction}

The theoretical and experimental investigation of the dynamic behavior of structural elements such as string, beams, plates and shells under influence of moving loads have been the subject of study of many researchers since last decades. Structures subjected to moving loads are often encountered in engineering practice. Some examples of such applications can be addressed as ropes of transporting systems, weapon firing barrels, overhead cranes, machining operations in milling and turning and plates used in road or airfield traveled by moving ground vehicles or aero vehicles, respectively. These structures may be exposed to much larger displacements than when they are under static loads. For small oscillations, the response of deformable bodies like plates can be suitably explained by linear equations. If the amplitude of oscillation increases then nonlinear effects appear. The cause of nonlinearity may be material, geometric and inertial in nature. For such cases, certainly accurate calculation of the dynamic response is necessary for reliable design and hence better performance. Most recent articles in this topic deal with the linear behavior of plates subjected to moving masses and forces whereas in reality such systems naturally have nonlinear behavior.

Many simple moving load problems and their analytical solutions were studied by Fryba (1999). The extensive studies of nonlinear dynamical characteristics of thin or thick beams under moving masses/forces were investigated in several works (Mamandi et al., 2010a,b, 2013; Mamandi and Kargarnovin, 2011a,b, 2013, 2014). A procedure incorporating the finite strip 
method together with a spring system was developed and applied to treat the response of rectangular plate structures resting on an elastic foundation due to moving accelerated loads by Huang and Thambiratnam (2001). The effect of initial moving velocity, acceleration and initial load position on the response was discussed. Wu (2003) studied dynamic response of a rectangular plate subjected to multiple forces moving along a circular path. A technique to predict dynamic responses of a two-dimensional rectangular plate traveled by a transverse moving line load using the one-dimensional equivalent beam model or the scale beams subjected to a moving concentrated load was presented by Wu (2005). The elastodynamic response of a rectangular Mindlin plate subjected to a distributed moving mass was investigated by Gbadeyan and Dada (2006). The set of governing PDEs of motion that include the effect of shear deformation and rotary inertia was expressed in a dimensionless form. A finite difference algorithm was employed to transform the differential equations into a set of linear algebraic equations. The dynamic response of the beam-slab type bridge deck under influence of moving loads was obtained applying the Hamilton principle with modal superposition by Law et al. (2007). Wu (2007) studied the linear dynamical response of an inclined plate subjected to moving loads and including also the effects of the inertial, Coriolis and centrifugal forces. It has been reported that the effects of Coriolis force and centrifugal force become more significant at higher speed of the moving mass. Ghafoori et al. (2010) used a semi-analytical method to calculate the dynamic response of a rectangular plate due to a moving oscillator. The dynamic response of angle-ply laminated composite plates traversed by a moving mass or a moving force was investigated by Ghafoori and Asghari (2010) using finite element method based on the first-order shear deformation theory. Similarly, Mohebpour et al. (2011) presented a finite element model based on the first order shear deformation theory to investigate the dynamic behavior of laminated composite plates traversed by a moving oscillator. A new finite element which can be used in the analysis of transverse vibrations of plates under a moving point mass was presented by Esen (2013). An eigen-function expansion method was employed to solve the constitutive equation of motion of a rectangular plate under various boundary conditions and the motion of a traveling mass. Vaseghi Amiri et al. (2013) studied the dynamic response of an undamped moderately thick plate with arbitrary boundary conditions under motion of a moving mass. The FSDT (first-order shear deformation plate theory or Mindlin plate theory) was selected as venue to derive the governing equations of motion. Combined application of the Ritz method, the Differential Quadrature (DQ) method and the Integral Quadrature (IQ) method to vibration problems of rectangular plates subjected to accelerated traveling masses was investigated by Eftekhari and Jafari (2012).

In the present study, the effect of geometric nonlinearity caused by stretching of the mid-plane of a rectangular plate with immovable simply supported on all edges and under motion of accelerated/decelerated moving mass/force on the plate dynamic responses is investigated. Based on Hamilton's principle, the nonlinear governing coupled PDEs of motion are derived and solved applying Glarkin's method using the Adam-Bashforth-Moulton integration method via the MATLAB solver package to obtain the dynamic response of the plate.

\section{Mathematical modeling}

\subsection{Problem statement}

In Fig. 1 an isotropic and homogenous elastic rectangular plate of sides $a$ and $b$ (length $a$ and width $b$ ) simply supported on all edges with density $\rho$, uniform thickness $h$, mass per unit area $\mu=\rho h$, modulus of elasticity $E$, Poisson's ratio $\nu$, bending stiffness $D=E h^{3} / 12\left(1-\nu^{2}\right)$ and subjected to a moving mass $m_{e}$ with velocity $V$ and constant acceleration/deceleration $A$ is shown. As can be seen in Fig. 1, the origin of the Cartesian coordinate system xoy is placed 
at the lower left corner of the plate. In our upcoming analysis it is assumed that the moving mass travels along a straight line at the middle of the plate width, i.e. $y=b / 2$ (see Fig. 1 ). It should be mentioned that in our upcoming analysis when the moving mass enters the left side of the plate at time $t=0$, zero initial conditions are assumed. Moreover, in our analysis it has been assumed that the moving mass during its travel never loses its contact with the plate surface under it. In this study, the nonlinear dynamic behavior for the coupled longitudinal and transversal in-plane and out of plane displacements of a uniform rectangular plate under the action of moving mass/force is considered. It is assumed that the damping behavior follows the viscous nature. Moreover, the plate deforms within the linear elastic range and, therefore, Hook's law is prevailing.
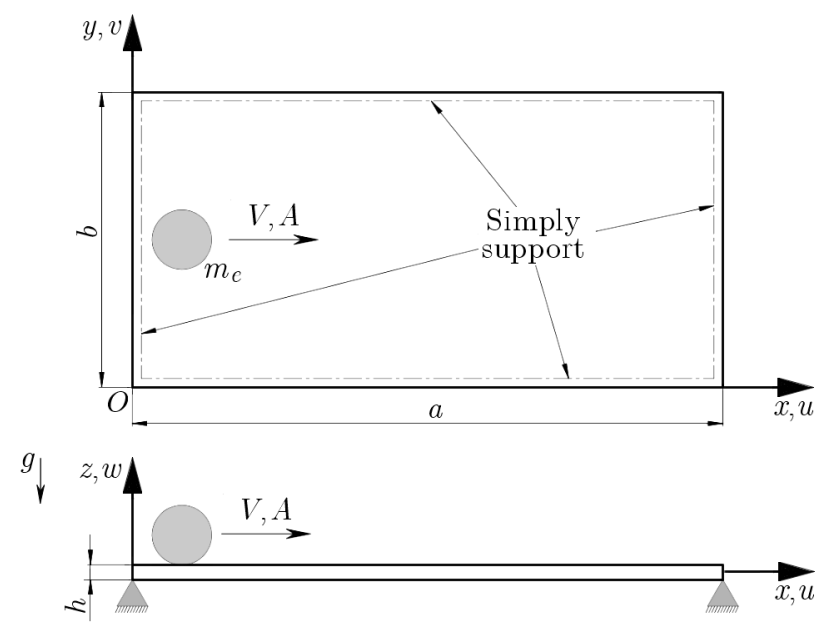

Fig. 1. A rectangular plate of sides $a$ and $b$ simply supported on all edges subjected to a traveling mass $m_{e}$ with velocity $V$ and constant acceleration/deceleration $A$

\subsection{Formulation}

According to the von-Karman nonlinear strain-displacement relations, the normal strains $\varepsilon_{x}$ and $\varepsilon_{y}$ and the shearing strain $\gamma_{x y}$ of the middle surface for the plate shown in Fig. 1 are expressed as follows (Nayfeh and Mook, 1995)

$$
\varepsilon_{x}=u_{, x}+\frac{1}{2} w_{, x}^{2} \quad \varepsilon_{y}=v_{, y}+\frac{1}{2} w_{, y}^{2} \quad \gamma_{x y}=u_{, y}+v_{, x}+w_{, x} w_{, y}
$$

in which $u(x, y, t), v(x, y, t)$ and $w(x, y, t)$ represent the time dependent displacements of an arbitrary point located on the middle surface of the plate in the $x, y$ and $z$ directions, respectively measured from the equilibrium position when unloaded. Also, in our notation the subscripts $(, x),(, y)$ and $(, t)$ stand for the derivative with respect to the spatial coordinates $x$ and $y$ and time $t$, respectively. To obtain the nonlinear governing differential equations of motion by applying Hamilton's principle, the kinetic energy $T$ of the rectangular plate under consideration is (Meirovitch, 1997; Nayfeh and Mook, 1995)

$$
T=\frac{1}{2} \rho h \int_{0}^{a} \int_{0}^{b}\left(u_{, t}^{2}+v_{, t}^{2}+w_{, t}^{2}\right) d x d y
$$

and according to Kirchhoff's plate hypothesis, the strain energy $U$ of the plate is given by (Meirovitch, 1997)

$$
U=\frac{1}{2} \int_{0}^{a} \int_{0}^{b} \int_{-h / 2}^{h / 2}\left(\sigma_{x} \varepsilon_{x}+\sigma_{y} \varepsilon_{y}+\tau_{x y} \gamma_{x y}\right) d x d y d z
$$


where $\sigma_{x}, \sigma_{y}$ and $\tau_{x y}$ are normal and shear in-plane stresses, respectively, and for the plate under consideration can be obtained by Hook's law given by (Timoshenko, 1959; Ugural, 1999)

$$
\sigma_{x}=\frac{E}{1-\nu^{2}}\left(\varepsilon_{x}+\nu \varepsilon_{y}\right) \quad \sigma_{y}=\frac{E}{1-\nu^{2}}\left(\varepsilon_{y}+\nu \varepsilon_{x}\right) \quad \tau_{x y}=\frac{E}{2(1+\nu)} \gamma_{x y}
$$

Now, we can establish the Lagrangian function of the system as: $L=T-\left(U-W_{e}\right)$. Applying Hamilton's principle on $L$, yields to (Meirovitch, 1997)

$$
\delta \int_{t_{1}}^{t_{2}} L d t=0 \Rightarrow \delta \int_{t_{1}}^{t_{2}}(U-T) d t=\int_{t_{1}}^{t_{2}} \delta W_{e} d t
$$

in which the total external virtual work done $\delta W_{e}$ by the gravity and the traveling mass acting on the plate at the location $x=x_{0}(t)$ and $y=y_{0}(t)=b / 2$ is (Meirovitch, 1997)

$$
\delta W_{e}=-\left.\int_{0}^{b} \int_{0}^{a} m_{e}\left(g+w_{, t t}+2 V w_{, x t}+V^{2} w_{, x x}+A w_{, x}\right) \delta w\right|_{x_{0}(t)=\frac{1}{2} A t^{2}+V t+x_{0}, y_{0}(t)=\frac{b}{2}} d x d y
$$

in which, $m_{e} w_{, t t}, 2 m_{e} V w_{, x t}, m_{e} V^{2} w_{, x x}$ and $A w_{, x}$ are inertial, Coriolis, centrifugal and acceleration/deceleration induced forces acting on the elastic surface of the plate, respectively due to motion of the mass.

After substitution of Eqs. (2.2), (2.3) and (2.6) into equation (2.5), performing integration and doing some mathematical simplifications one would get the nonlinear governing coupled PDEs of motion (EOMs) as follows:

— the force relation in the $x$ direction

$$
u_{, x x}+w_{, x} w_{, x x}+\nu\left(v_{, x y}+w_{, y} w_{, x y}\right)+\frac{1}{2}(1-\nu)\left(u_{, y y}+v_{, x y}+w_{, x} w_{, y y}+w_{, y} w_{, x y}\right)=\frac{1}{c_{p}^{2}} u_{, t t}
$$

— the force relation in the $y$ direction

$$
u_{, y y}+w_{, y} w_{, y y}+\nu\left(u_{, x y}+w_{, x} w_{, x y}\right)+\frac{1}{2}(1-\nu)\left(u_{, x y}+v_{, x x}+w_{, x} w_{, x y}+w_{, y} w_{, x x}\right)=\frac{1}{c_{p}^{2}} v_{, t t}
$$

— the force relation in the $z$ direction

$$
\begin{aligned}
\frac{1}{12} h^{2} \nabla^{4} w-u_{, x} w_{, x x}-\frac{1}{2} w_{, x}^{2} w_{, x x}-v_{, y} w_{, y y}-\frac{1}{2} w_{, y}^{2} w_{, y y} \\
-\nu\left(v_{, y} w_{, x x}+\frac{1}{2} w_{, y}^{2} w_{, x x}+u_{, x} w_{, y y}+\frac{1}{2} w_{, x}^{2} w_{, y y}\right) \\
-(1-\nu)\left(u_{, y} w_{, x y}+v_{, x} w_{, x y}+w_{, x} w_{, y} w_{, x y}\right)+\frac{c}{c_{p}^{2} \rho h} w_{, t} \\
=\frac{1}{c_{p}^{2}}\left(w_{, x} u_{, t t}+w_{, y} v_{, t t}-w_{, t t}\right)-\frac{m_{e}}{c_{p}^{2} \rho h} \delta\left(x-x_{0}(t)\right) \delta\left(y-y_{0}(t)\right) \\
\left.\cdot\left(w_{, x x} V^{2}+w_{, t t}+2 w_{, x t} V+w_{, x} A+g\right)\right|_{x_{0}(t)=\frac{1}{2} A t^{2}+V t+x_{0}, y_{0}(t)=\frac{b}{2}}
\end{aligned}
$$

in which $c_{p}^{2}=E /\left[\rho\left(1-\nu^{2}\right)\right]$ and operator $\nabla^{4}=\left(\frac{\partial^{4}}{\partial x^{4}}+2 \frac{\partial^{4}}{\partial x^{2} \partial y^{2}}+\frac{\partial^{4}}{\partial y^{4}}\right)$. Furthermore, $\delta\left(x-x_{0}(t)\right) \delta\left(y-y_{0}(t)\right)$ represents two dimensional Dirac's delta function in which $x_{0}(t)$ and $y_{0}(t)$ are the instantaneous positions of the moving mass traveling on the plate. In the case the mass is traveling with velocity $V$ and constant acceleration/deceleration $A$ on a straight path along the trajectory parallel to the side $a$ at the middle of the plate width, i.e. $y=b / 2$, then its 
instantaneous position is given by $x_{0}(t)=A t^{2} / 2+V t+x_{0}$ and $y_{0}(t)=b / 2$, where $x_{0}$ represents the initial position of the mass at the start of its motion. In addition, $c$ (or $c_{m n}$ ) coefficient is internal viscous damping of the plate related to modal damping ratio, namely $\zeta_{m n}$ expressed by $\zeta_{m n}=c_{m n} /\left[2\left(\lambda_{m n} \omega_{m n}\right)\right]$ (Amabili, 2004), where $\omega_{m n}$ is the natural frequency of the $m$ th- $n$th mode of vibration and $\lambda_{m n}$ is the modal mass of this mode given by $\lambda_{m n}=\rho h a b / 4$ (Amabili, 2004).

\section{Solution method}

In this study, Galerkin's method is chosen as a powerful mathematical tool to analyze vibrations of the plate. Based on the separation of variables technique, the response of the plate in terms of the linear free-oscillation modes can be assumed as follows (Amabili, 2004):

$$
\begin{aligned}
& u(x, y, t)=\sum_{i}^{m} \sum_{j}^{n} p_{i j}(t) \phi_{i j}(x, y)=P(t) \boldsymbol{\Phi}^{\mathrm{T}}(x, y) \\
& v(x, y, t)=\sum_{k}^{n} \sum_{l}^{m} q_{k l}(t) \psi_{k l}(x, y)=Q(t) \boldsymbol{\Psi}^{\mathrm{T}}(x, y) \\
& w(x, y, t)=\sum_{v}^{M} \sum_{z}^{N} r_{v z}(t) \theta_{v z}(x, y)=R(t) \boldsymbol{\Theta}^{\mathrm{T}}(x, y)
\end{aligned}
$$

where $P(t), Q(t)$ and $R(t)$ are vectors listing the generalized coordinate $p_{i j}(t), q_{k l}(t)$ and $r_{v z}(t)$, respectively, and $\phi(x, y), \boldsymbol{\Psi}(x, y)$ and $\boldsymbol{\Theta}(x, y)$ are some vectorial functions collecting the first mode shapes (eigen-functions) of $\phi_{i j}(x, y), \psi_{k l}(x, y)$ and $\theta_{v z}(x, y)$, respectively. In the next step, primarily we substitute Eqs. (3.1) into Eqs. (2.7), (2.8) and (2.9), then on the resulting relations, pre-multiplying both sides of Eq. $(2.7)$ by $\boldsymbol{\Phi}^{\mathrm{T}}(x, y)$, Eq. $(2.8)$ by $\boldsymbol{\Psi}^{\mathrm{T}}(x, y)$ and Eq. (2.9) by $\Theta^{\mathrm{T}}(x, y)$, integrating over the interval $(0, a)$ and $(0, b)$ and imposing the orthogonality property of the vibration modes of the plate along with the properties of the two dimensional Dirac delta function, the resulting nonlinear coupled modal ODEs of motion in matrix form are as follows $(i=l=2,4, \ldots, m, j=k=1,2, \ldots, n, v=1,2, \ldots, M, z=1,2, \ldots, N)$

$$
\begin{aligned}
& \frac{1}{c_{p}^{2}} \sum_{i, j}^{m, n} I_{7, i j} \ddot{p}_{i j}(t)-\sum_{i, j}^{m, n}\left[I_{1, i j}+\frac{1}{2}(1-\nu) I_{5, i j}\right] p_{i j}(t)-\frac{1}{2}(1+\nu) \sum_{k, l}^{n, m} \sum_{i, j}^{m, n} I_{3, k l i j} q_{k l}(t) \\
& \quad-\sum_{v, z}^{M, N} \sum_{i, j}^{m, n}\left[I_{2, v z i j}+\frac{1}{2}(1+\nu) I_{4, v z i j}+\frac{1}{2}(1-\nu) I_{6, v z i j}\right] r_{v z}^{2}(t)=0 \\
& \frac{1}{c_{p}^{2}} \sum_{k, l}^{n, m} I_{14, k l} \ddot{q}_{k l}(t)-\sum_{k, l}^{n, m}\left[I_{8, k l}+\frac{1}{2}(1-\nu) I_{12, k l}\right] q_{k l}(t)-\frac{1}{2}(1+\nu) \sum_{i, j}^{m} \sum_{k, l}^{m, n} I_{10, i j k l} p_{i j}(t) \\
& \quad-\sum_{v, z}^{M, N} \sum_{k, l}^{n, m}\left[I_{9, v z k l}+\frac{1}{2}(1+\nu) I_{11, v z k l}+\frac{1}{2}(1-\nu) I_{13, v z k l}\right] r_{v z}^{2}(t)=0 \\
& \sum_{v, z}^{M, N}\left(\frac{m_{e}}{c_{p}^{2} \rho h} I_{34, v z}+\frac{1}{c_{p}^{2}} I_{31, v z}\right) \ddot{r}_{v z}(t)+\sum_{v, z}^{M, N}\left(2 m_{e} V I_{35, v z}+\frac{c_{v z}}{c_{p}^{2} \rho h} I_{31, v z}\right) \dot{r}_{v z}(t) \\
& \quad+\sum_{v, z}^{M, N}\left[\frac{h^{2}}{12}\left(I_{15, v z}+2 I_{16, v z}+I_{17, v z}\right)+\frac{m_{e} V^{2}}{c_{p}^{2} \rho h} I_{33, v z}+\frac{m_{e} A}{c_{p}^{2} \rho h} I_{35, v z}\right] r_{v z}(t) \\
& \quad-\sum_{v, z}^{M, N}\left\{\frac{1}{2}\left[I_{19, v z}+I_{21, v z}+\nu\left(I_{23, v z}+I_{25, v z}\right)\right]+(1-\nu) I_{28, v z}\right\} r_{v z}^{3}(t)
\end{aligned}
$$




$$
\begin{aligned}
& +\sum_{i, j}^{m, n} \sum_{v, z}^{M, N}\left\{\left[I_{18, i j v z}+\nu I_{24, i j v z}+(1-\nu) I_{26, i j v z}\right] p_{i j}(t)+\frac{1}{c_{p}^{2}} I_{29, i j v z} \ddot{p}_{i j}(t)\right\} r_{v z}(t) \\
& +\sum_{k, l}^{n, m} \sum_{v, z}^{M, N}\left\{\left[I_{20, k l v z}+\nu I_{22, k l v z}+(1-\nu) I_{27, k l v z}\right] q_{k l}(t)+\frac{1}{c_{p}^{2}} I_{30, k l v z} \ddot{q}_{r s}(t)\right\} r_{v z}(t) \\
& =\frac{m_{e} g}{c_{p}^{2} \rho h} \sum_{v, z}^{M, N} I_{32, v z}
\end{aligned}
$$

in which the dot mark over any parameter indicates the derivative with respect to time, i.e., t. All matrices $I_{1}$ to $I_{35}$ appearing in the above relations are given in Appendix A. It is clear that Eqs. (3.2) are three nonlinear coupled second-order ordinary differential equations (ODEs). The boundary conditions for a plate with immovable simple supports on all edges are (Timoshenko, 1959; Ugural, 1999):

- essential BCs

$$
\begin{aligned}
& u=v=w=0 \quad \text { at } \quad x=0, \quad x=a \\
& u=v=w=0 \quad \text { at } \quad y=0, \quad y=b
\end{aligned}
$$

- natural BCs

$$
\begin{aligned}
M_{x} & =0 \rightarrow w_{, x x}=0 \\
M_{y}=0 & \rightarrow w_{, y y}=0
\end{aligned} \quad \text { at } \quad \begin{array}{lll}
x=0, & x=a \\
\text { at } & y=0, & y=b
\end{array}
$$

Moreover, the initial conditions (ICs) for the plate are

ICs: $\quad u(x, y, 0)=u_{, t}(x, y, 0)=v(x, y, 0)=v_{, t}(x, y, 0)=w(x, y, 0)=w_{, t}(x, y, 0)=0$

The equations of motion for a plate subjected to an equivalent concentrated moving force $F$ of magnitude $m_{e} g$ can be derived from the equations of motion for a plate subjected to a moving mass by neglecting the inertial effect of the traveling mass. For this system, Eqs. (2.9) and (3.2) 3 are rewritten as follows $(i=l=2,4, \ldots, m, j=k=1,2, \ldots, n, v=1,2, \ldots, M, z=1,2, \ldots, N)$

$$
\begin{aligned}
& \frac{1}{12} h^{2} \nabla^{4} w-u_{, x} w_{, x x}-\frac{1}{2} w_{, x}^{2} w_{, x x}-v_{, y} w_{, y y}-\frac{1}{2} w_{, y}^{2} w_{, y y} \\
& \quad-\nu\left(v_{, y} w_{, x x}+\frac{1}{2} w_{, y}^{2} w_{, x x}+u_{, x} w_{, y y}+\frac{1}{2} w_{, x}^{2} w_{, y y}\right) \\
& \quad-(1-\nu)\left(u_{, y} w_{, x y}+v_{, x} w_{, x y}+w_{, x} w_{, y} w_{, x y}\right)+\frac{c}{c_{p}^{2} \rho h} w_{, t} \\
& \quad=\frac{1}{c_{p}^{2}}\left(w_{, x} u_{, t t}+w_{, y} v_{, t t}-w_{, t t}\right)-\left.\frac{m_{e} g}{c_{p}^{2} \rho h} \delta\left(x-x_{0}(t)\right) \delta\left(y-y_{0}(t)\right)\right|_{x_{0}(t)=\frac{1}{2} A t^{2}+V t+x_{0}, y_{0}(t)=\frac{b}{2}} \\
& \sum_{v, z} \frac{1}{c_{p}^{2}} I_{31, v z} \ddot{r}_{v z}(t)+\sum_{v, z}^{M, N} \frac{c_{v z}}{c_{p}^{2} \rho h} I_{31, v z} \dot{r}_{v z}(t)+\sum_{v, z} \frac{h^{2}}{12}\left(I_{15, v z}+2 I_{16, v z}+I_{17, v z}\right) r_{v z}(t) \\
& \quad-\sum_{v, z}^{M, N}\left\{\frac{1}{2}\left[I_{19, v z}+I_{21, v z}+\nu\left(I_{23, v z}+I_{25, v z}\right)\right]+(1-\nu) I_{28, v z}\right\} r_{v z}^{3}(t) \\
& \quad+\sum_{i, j}^{m, n} \sum_{v, z}^{M, N}\left\{\left[I_{18, i j v z}+\nu I_{24, i j v z}+(1-\nu) I_{26, i j v z}\right] p_{i j}(t)+\frac{1}{c_{p}^{2}} I_{29, i j v z} \ddot{p}_{i j}(t)\right\} r_{v z}(t) \\
& \quad+\sum_{k, l}^{n, m} \sum_{v, z}^{M, N}\left\{\left[I_{20, k l v z}+\nu I_{22, k l v z}+(1-\nu) I_{27, k l v z}\right] q_{k l}(t)+\frac{1}{c_{p}^{2}} I_{30, k l v z} \ddot{q}_{r s}(t)\right\} r_{v z}(t) \\
&= \frac{m_{e} g}{c_{p}^{2} \rho h} \sum_{v, z}^{M, N} I_{32, v z}
\end{aligned}
$$


where Eqs. (2.7), (2.8), (3.2) 1 and (3.2) 2 remain unchanged.

In order to solve Eqs. (3.2), all entries in the matrices listed in Appendix A should be calculated. It can be seen that the following functions (mode shapes) for $\phi_{i j}(x, y), \psi_{k l}(x, y)$ and $\theta_{v z}(x, y)$ will satisfy both the linearized equations of motion and boundary conditions of the plate with immovable simpe supports on all edges (Vaseghi et al., 2013)

$$
\begin{aligned}
\phi_{i j}(x, y)=\sin \frac{i \pi x}{a} \sin \frac{j \pi y}{b} & i=2,4, \ldots, m, & j=1,2, \ldots, n \\
\psi_{k l}(x, y)=\sin \frac{k \pi x}{a} \sin \frac{l \pi y}{b} & k=1,2, \ldots, n, & l=2,4, \ldots, m \\
\theta_{v z}(x, y)=\sin \frac{v \pi x}{a} \sin \frac{z \pi y}{b} & v=1,2, \ldots, M, & z=1,2, \ldots, N
\end{aligned}
$$

Now, we use Eq. (3.7) to calculate all matrix quantities given in Appendix A. In the next step, these evaluated matrices will be used in Eqs. (3.2), and later, the set of equations will be solved numerically using the Adams-Bashforth-Moulton integration method via MATLAB solver package to obtain values of $p_{i j}(t), q_{k l}(t)$ and $r_{v z}(t)$. By back substitution of $p_{i j}(t), q_{k l}(t)$ and $r_{v z}(t)$ into Eqs. (2.7) to (2.9), $u(x, y, t), v(x, y, t)$ and $w(x, y, t)$ can be obtained, respectively. Subsequently, after obtaining values for $u(x, y, t), v(x, y, t)$ and $w(x, y, t)$ the dynamic response of the rectangular plate under the effect of three types of mass motion: (a) accelerating, (b) decelerating and (c) constant velocity motion are obtained. The obtained results for the plate response under each of those three types of mass/force motions are shown separately in Figs. 4 to 6 . The detailed kinematical discussions of the above different motions are described below (Mamandi et al., 2010a, 2013):

(a) In the case of constant accelerating type of motion $\left(x_{0}(t)=0.5 A_{a c} t^{2}+V_{0} t+x_{0}\right.$, $A_{a c}=$ const $>0$ ), it is assumed that the plate is at rest when the mass $m_{e}$ enters the plate at $x_{0}=0$ and $t_{0}=0$, and with initial velocity $V_{0}=0$ and it arrives at the other end of the plate, i.e. $x=a$ with final velocity $V$. The total traveling time in the plate span $t_{1}$ and mass exit velocity $V$ will be: $t_{1}=2 a / V, V=\sqrt{2 A_{a c} a}$.

(b) In the case of constant decelerating type of motion $\left(x_{0}(t)=0.5 A_{d e} t^{2}+V_{0} t+x_{0}\right.$, $A_{d e}=$ const $\left.<0\right)$, it is also assumed that the plate is at rest when the mass $m_{e}$ enters the plate at $x_{0}=0$ and $t_{0}=0$ and with entrance velocity $V_{0}$ (non-zero initial velocity), and it stops $(V=0)$ at the other end of the plate, i.e. $x=a$. The total traveling time in the plate span $t_{2}$ and mass entrance velocity $V_{0}$ will be: $t_{2}=2 a / V_{0}, V_{0}=\sqrt{2\left|A_{d e}\right| a}$.

(c) In the case of uniform velocity type of motion $\left(x_{0}(t)=V t+x_{0}\right)$, it is also assumed that the plate is at rest when the mass $m_{e}$ enters the plate at $x_{0}=0$ and $t_{0}=0$ with mass constant velocity $V$, and it reaches the other end of the plate, i.e. $x=a$ at the instant $t_{3}$. The total traveling time in the plate span will be: $t_{3}=a / V$.

\section{Verification of the results and case studies}

As mentioned in the introduction, at the moment no specific results exist for the problem under consideration in the literature. Therefore, to verify the validity of the results obtained in this study, we primarily consider some special cases by which our results can be compared with those existing in the literature.

\subsection{Verification of the results in linear analysis}

In the first attempt, we neglect the higher order terms in Eqs. (2.7) to (2.9) and structural damping for the plate, i.e., $c=0$ and $A=0$. This will lead us to a set of new relations for 
$u(x, y, t), v(x, y, t)$ and $w(x, y, t)$ representing the linear form of governing EOMs of a plate subjected to a moving load with constant velocity $V$. By doing this, Eq. (2.9) is decoupled from Eqs. (2.7) and (2.8) and transformed into a linear form as follows (Leissa, 1969)

$$
\nabla^{4} w+\frac{\rho h}{D} w_{, t t}=-\left.\frac{F}{D} \delta\left(x-x_{0}(t)\right) \delta\left(y-y_{0}(t)\right)\right|_{x_{0}(t)=V t, y_{0}(t)=\frac{b}{2}}
$$

To verify the validity of the obtained results out of our analysis, we consider a simply supported rectangular plate traveled by a moving force $F$ with the data given by Eftekhari and Jafari (2012): $a=b=10 \mathrm{~m}, E=200 \cdot 10^{9} \mathrm{~Pa}, \rho=7850 \mathrm{~kg} / \mathrm{m}^{3}, F=3.13 \mathrm{~N}, \rho h / D=0.0001 \mathrm{~kg} /\left(\mathrm{N} \mathrm{m}^{3}\right)$, $y_{0}(t)=b / 2, V=5 \mathrm{~m} / \mathrm{s}, \nu=0.3, c=0 \mathrm{Ns} / \mathrm{m}^{2}$ and $A=0$. By employing Eqs. (4.1) and (3.6) and based on the above data, the computer code was run for this case and the variation of vertical dynamic displacements $w$ of the central point of the plate vs. time $t$ were calculated. The outcome results are depicted and compared with other existing results in Fig. 2. A close inspection of the curves in Fig. 2 indicates very good agreements between the two outcome results.

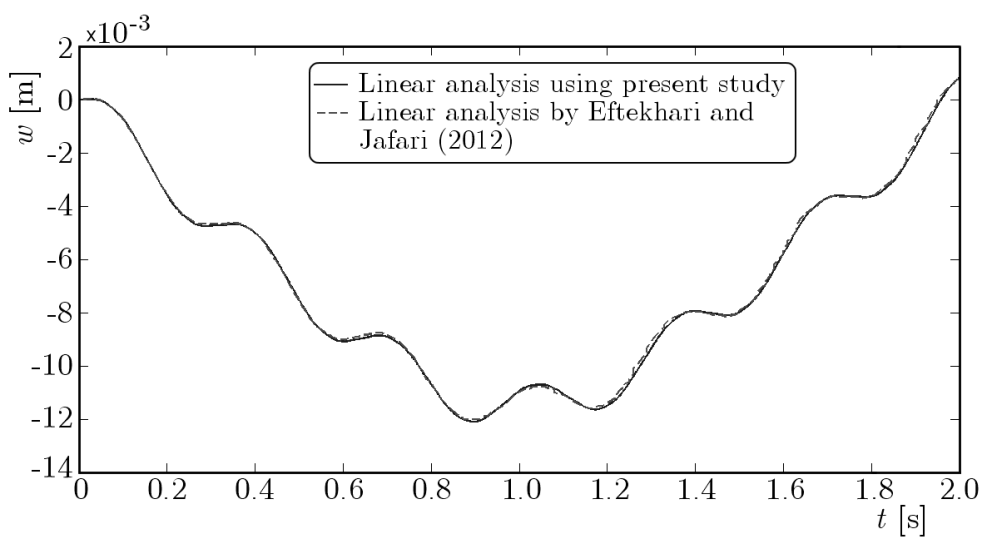

Fig. 2. Time history for the vertical displacement of the central point of the simply support plate subjected to the moving force $F$

\subsection{Verification of the results in nonlinear analysis}

As described earlier, in this study, to extend checking on the validity of our obtained results we prepared appropriate APDL (ANSYS Parametric Design Language) routine in the environment of ANSYS software to simulate the response of the moving force on the plate. Then, the linear and nonlinear FEM solutions have been compared with those obtained by the linear and nonlinear analytical solutions applying the mode summation technique. In the modeling of the plate, we used shell63 element defined in this software which is suitable for analyzing shell type structures. This element is a 3-D 4-noded shell element having 6 DOFs with 3 -translational DOF in the $x, y$ and $z$ directions and 3-rotational DOF in each node about above the mentioned axes. Moreover, this element is adopted to exert both in-plane and out of plane (normal) loads and suitable for large deflections. Now, to establish our calculations, we consider a plate with geometry and mechanical properties listed as: $a=4 \mathrm{~m}, b=2 \mathrm{~m}, h=0.01 \mathrm{~m}, E=200 \cdot 10^{9} \mathrm{~Pa}$, $\rho=7850 \mathrm{~kg} / \mathrm{m}^{3}, g=9.81 \mathrm{~m} / \mathrm{s}^{2}$ and $\nu=0.3$.

Figure 3 illustrates the variation of the central point vertical deflection $w[\mathrm{~m}]$ of the plate with all edges simply supported using the above mentioned data vs. time $t$ at velocity ratio of $\alpha=1$ for the traveling force of $F=0.25 \mu \mathrm{gab}[\mathrm{N}]$ under influence of constant velocity motion using FEM and analytical analysis, respectively. From this figure, one can conclude that the results for the plate central point vertical dynamic displacement obtained by FEM and analytical solutions using nonlinear or linear analysis are almost the same which shows very good agreement between 
these analytical results obtained via the mode summation technique and FEM analysis. The suitable number of elements which has been used for the plate to converge the linear/nonlinear results is 288 elements with 24 elements used in the length $a$ and 12 elements used in the width $b$ of the plate. It should be mentioned that the maximum relative difference between the obtained results for the plate central point vertical deflection using linear and FEM solutions is $4.8 \%$ at the instant $0.073 \mathrm{~s}$, and also the maximum relative difference between the obtained results using nonlinear analysis and FEM solution is $1 \%$ at the instant $0.09 \mathrm{~s}$.

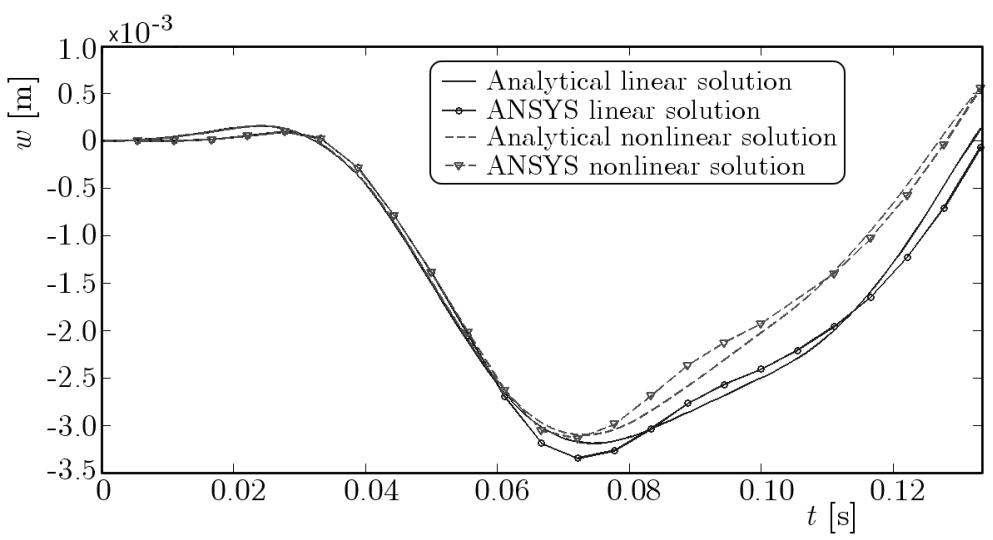

Fig. 3. Variation of the vertical deflection of the central point $w[\mathrm{~m}]$ vs. time $t[\mathrm{~s}]$ for the simply supported plate affected by the moving force $F=0.25 \mu \mathrm{gab}[\mathrm{N}]$ under constant velocity motion at $\alpha=1$ using analytical and FEM analysis for linear and nonlinear solutions

\section{Results and discussions}

After being satisfied about the validity of the solution technique, the plate central point instantaneous dynamic vertical deflection is calculated in the next step. In obtaining these results, a steel plate with the same specifications as mentioned above in Section 4.2, but in addition having $\zeta=0.033$ (Mamandi et al., 2010a,b) is considered.

It should be mentioned that all deflection variations vs. moving mass instantaneous position are given in a non-dimensional form that is $w_{\max } / w_{0}$. Moreover, it has to be pointed out that based on the conducted convergence study related to the linear and nonlinear analyses, 9 modes of vibration are taken into account for steady state answers for $u(x, y, t), v(x, y, t)$ and $w(x, y, t)$.

To clarify the results and in order to have a better insight into interpreting the variation of the obtained results, we tried to present the results in dimensionless forms. So, we begin with defining the normalized maximum dynamic vertical deflection of the central point of the plate to its maximum static response at the same point. The static downward deflection of the plate central point under a concentrated mass applied at the same point is equal to $w_{0}=0.01651 m_{e} g a^{2} / D$ (Timoshenko, 1959). Moreover let us define the velocity ratio as $\alpha=T_{1} / T=V / V_{p}$ in which $V_{p}=a / T_{1}=\left(\omega_{n} a\right) /(2 \pi)$, where $T_{1}, T$ and $V_{p}$ denote the first natural period (fundamental period of transverse motion) of the plate, the total time taken by the moving load to cross from one side to the opposite side of the plate and the velocity of the reference load that would take the time of $T_{1}$ to traverse the plate of length $a$, respectively. Moreover, $\omega_{n}$ is the natural frequency of the plate given by (Leissa, 1969)

$$
\omega_{n}=\omega_{i j}=\pi^{2}\left[\left(\frac{i}{a}\right)^{2}+\left(\frac{j}{b}\right)^{2}\right] \sqrt{\frac{D}{\rho a b}} \quad i, j=1,2, \ldots, n
$$

It should be pointed out that in conjuncture with the stretching effect of the mid-plane of the plate, the geometric nonlinearity behavior of the plate also depends on the ratio of the weight of 
the moving mass as well as amplitude of the equivalent concentrated moving force to the plate weight. For sure, we have done this study before any further calculation. It has been noticed that when this ratio is usually greater than 0.1 , the nonlinear geometric effects come into play.

Figures 4 and 5 show the variation of the dimensionless dynamic vertical deflection $\left(w_{\max } / w_{0}\right)$ vs. dimensionless time $t / t_{i}(i=1,2,3)$ at the central point of the simply supported rectangular plate traversed by the moving mass of $m_{e}=0.25 \mu a b[\mathrm{~kg}]$ and an equivalent concentrated force of $F=0.25 \mu \mathrm{gab}[\mathrm{N}]$, respectively with different velocity ratios $(\alpha=1,2,3,4)$ under the influence of three types of motion using linear and nonlinear solutions, respectively. As can
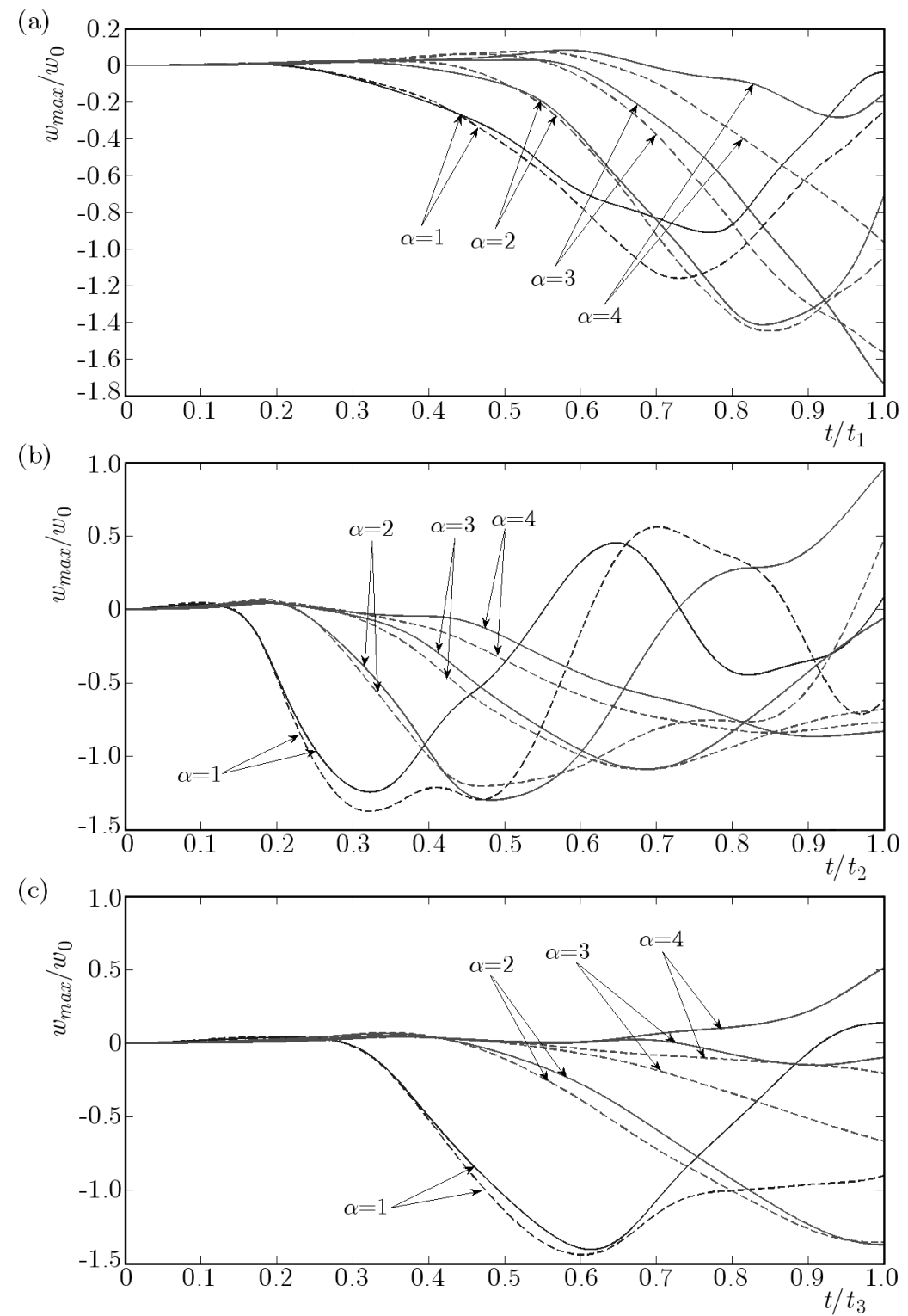

Fig. 4. Variation of the dimensionless dynamic vertical deflection $\left(w_{\max } / w_{0}\right)$ at the central point of the plate vs. normalized time $\left(t / t_{i}\right)$ for the simply supported rectangular plate traversed by the moving mass of $m_{e}=0.25 \mu a b[\mathrm{~kg}]$ with different velocity ratios $(\alpha=1,2,3,4)$ under influence of three types of motion; (a) accelerated motion, (b) decelerated motion, (c) uniform velocity motion; (-) nonlinear analysis, (- - ) linear analysis

be seen from Figs. 4 and 5, in the accelerating and decelerating types of motion by increasing the velocity ratio $\alpha$ up to $\alpha=3$ and 2 , respectively, the value of maximum nonlinear dynamic downward deflection increases and the reverse trend prevails afterwards, whereas in uniform ve- 

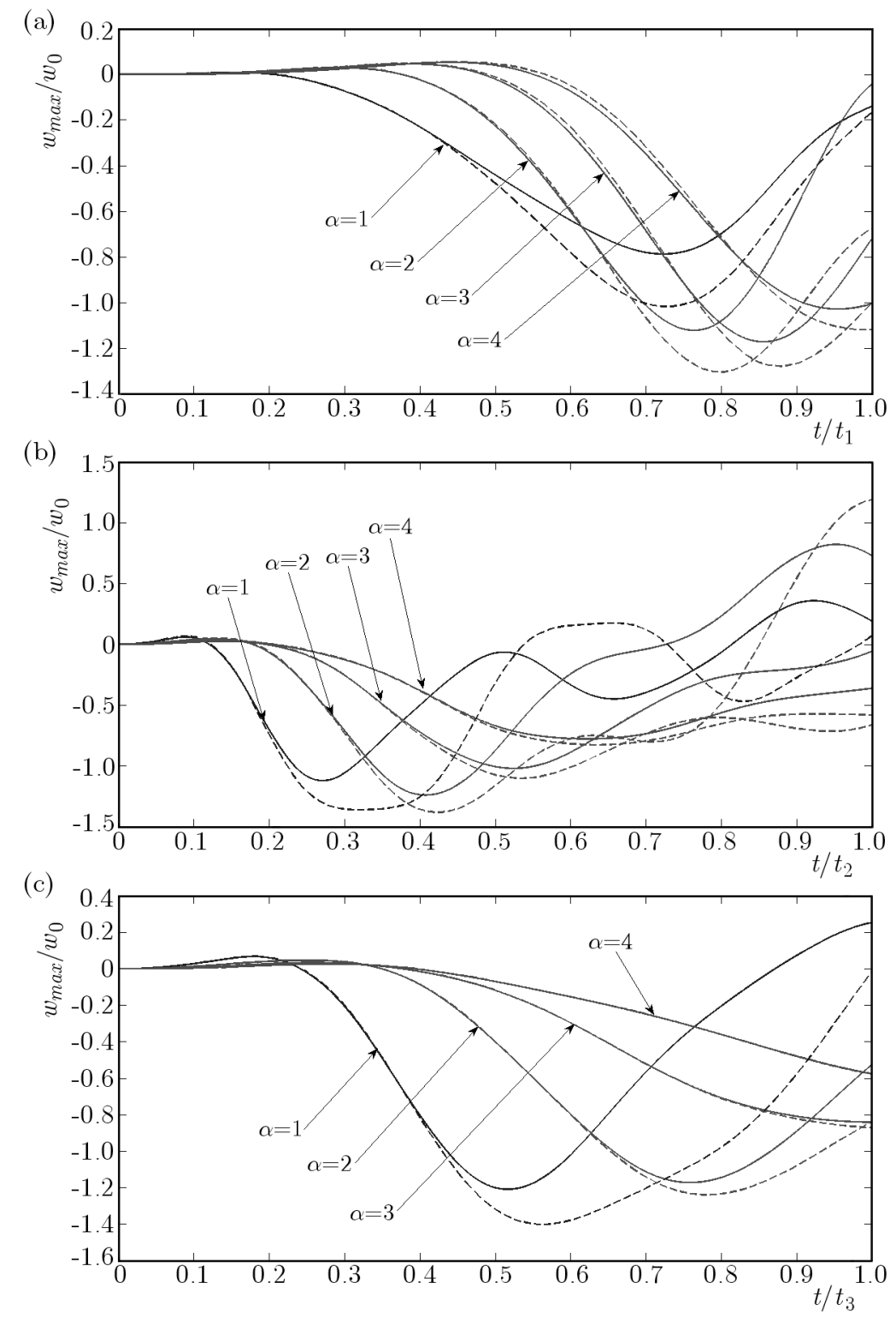

Fig. 5. Variation of the dimensionless dynamic vertical deflection $\left(w_{\max } / w_{0}\right)$ at the central point of the plate vs. normalized time $\left(t / t_{i}\right)$ for the simply supported rectangular plate traversed by the moving force of $F=0.25 \mu \mathrm{gab}[\mathrm{N}]$ with different velocity ratios $(\alpha=1,2,3,4)$ under influence of three types of motion; (a) accelerated motion, (b) decelerated motion, (c) uniform velocity motion;

(-) nonlinear analysis, (- - ) linear analysis

locity motion the reduction trend of the maximum dynamic downward deflection is always seen no matter what type of analysis is used. It is noticed that in the accelerating type of motion, the maximum dynamic deflection is reached at a much later time than the other two cases. Moreover, in the decelerating type of motion, the range of variation of the maximum dynamic deflection is larger with respect to the other two types of motion. Also, it can be seen from Figs. 4 and 5 that for a higher velocity ratio, i.e. $\alpha=4$, in uniform velocity motion, the vertical dynamic displacement of the plate central point yields a smaller value at the time of leaving the plate, which means the plate does not have enough time to respond accordingly against the fast speed of the moving mass/force. In addition, from these figures, it can be seen that in the decelerating type of motion in lower velocity ratios, i.e. $\alpha=1$ and 2 , there is a reverse (upward) displacement for the central point which occurs usually when the load leaves the plate. It can be 


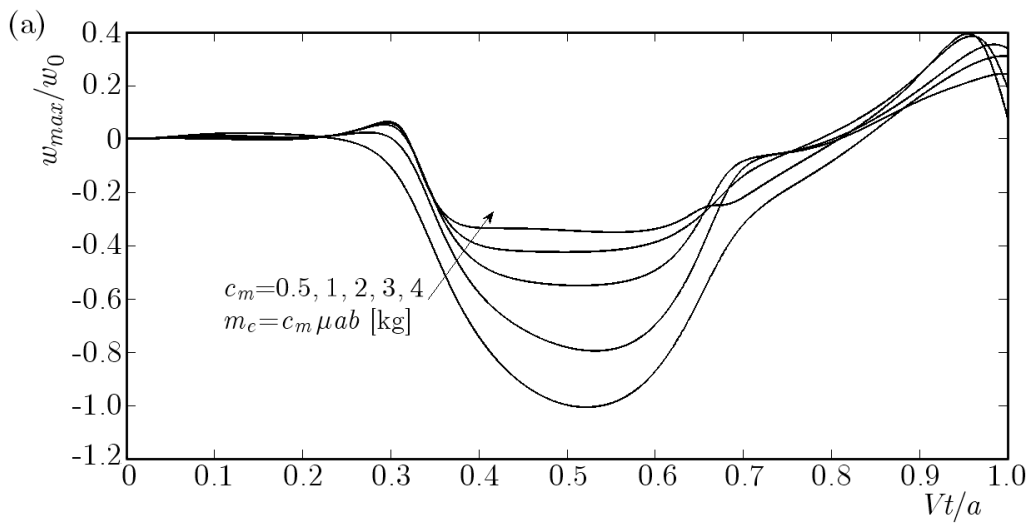

(b)

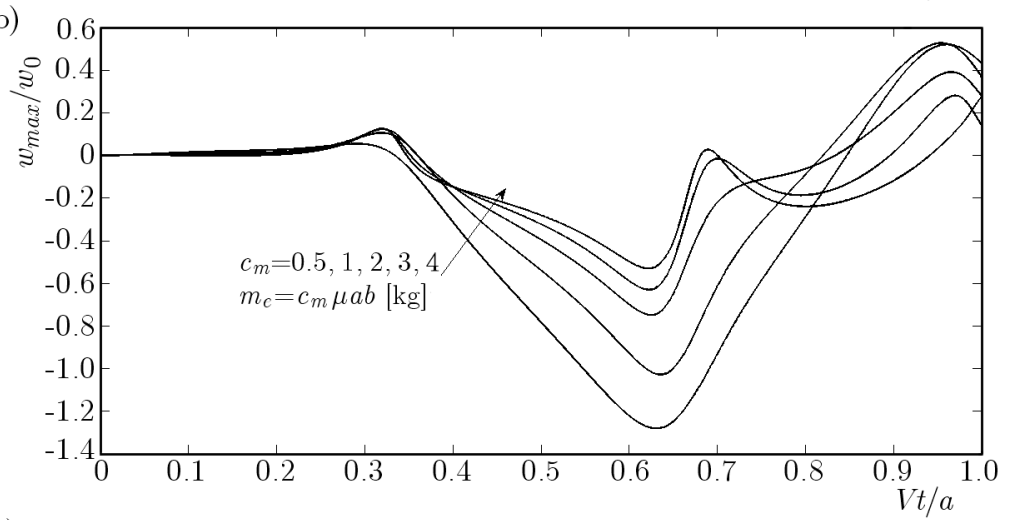

(c)
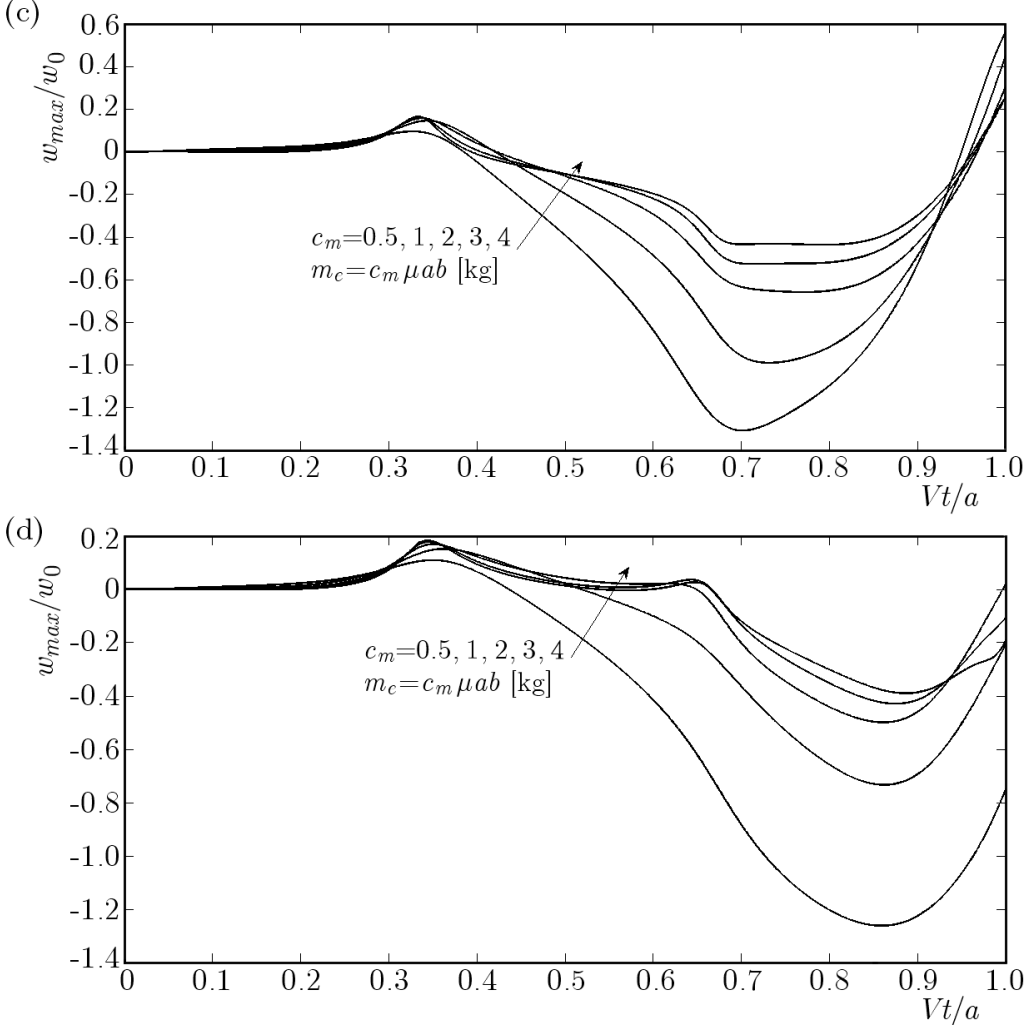

Fig. 6. Variation of the dimensionless dynamic vertical deflection $\left(w_{\max } / w_{0}\right)$ at the central point of the plate vs. normalized time $(V t / a)$ for the simply supported rectangular plate traversed by the moving mass of $m_{e}$ for different velocity ratios in the constant velocity type of motion; (a) $\alpha=0.75$, (b) $\alpha=1$,

(c) $\alpha=1.25$, (d) $\alpha=1.5$ 
observed in Figs. 4 and 5 that the obtained results from nonlinear solution have almost smaller values that those calculated by linear solution. In addition, from Figs. 4 and 5, it is concluded that the maximum difference for the plate central point deflection between nonlinear and linear solutions happens primarily in the decelerating motion and with a smaller difference in the case of uniform velocity and then in the accelerating type of motion, respectively.

To study the effect of weight of the moving mass to generate the nonlinearity behavior of the plate, Fig. 6 shows the variation of the dimensionless dynamic vertical deflection $\left(w_{\max } / w_{0}\right)$ of the simply supported rectangular plate for different velocity ratios $(\alpha=0.75,1,1.25$ and 1.5) traversed by different moving masses $m_{e}\left(m_{e}=c_{m} \mu a b[\mathrm{~kg}], c_{m}=0.5,1,2,3\right.$ and 4$)$ vs. the normalized instantaneous mass position, i.e. $V t / a$ using nonlinear analysis in the constant velocity type of motion. It can be seen from this figure that when $\alpha$ increases up to the velocity ratio $\alpha=1.25$, the maximum value of instantaneous dynamic deflection increases and decreases afterwards. The maximum dynamic deflection for all cases in this figure occurs at $m_{e}=0.5 \mu a b[\mathrm{~kg}]$ at $\alpha=1.25$.

\section{Conclusions}

Three nonlinear coupled partial differential equations of motion for the in-plane and out-of-plane displacements of a rectangular plate subjected to an accelerated/decelerated traveling mass as well as an equivalent concentrated force are solved, and the results are following:

- It can be seen that in the accelerating type of motion, the maximum dynamic deflection is reached at a much later time than in the other two types of motion.

- It is concluded that in the decelerating type of motion, the range of variation of the maximum dynamic deflection is larger with respect to the other two types of motion.

- For higher velocity ratios in the uniform velocity motion, the vertical dynamic displacement of the plate central point yields a smaller value at the time of leaving the plate.

- It is seen that in the moving mass/force problem in three types of motion, the obtained results by nonlinear solution have almost smaller values than those calculated by linear solution.

- It is observed that in conjuncture with the stretching effect of the mid-plane of the plate when the ratio of the weight of the moving mass as well as the amplitude of the equivalent concentrated moving force to the plate weight is usually greater than 0.1 , the geometric nonlinearity behavior of the plate comes into play.

- It is concluded that the maximum difference for the plate central point deflection between nonlinear and linear solutions happens primarily in the decelerating motion and with a smaller difference in the case of uniform velocity and then in the accelerating type of motion, respectively.

\section{Appendix A}

The definition of different matrices used in calculation of the nonlinear coupled ODEs of modal relations Eqs. (3.2) are

$$
\begin{array}{ll}
I_{1, i j}=\int_{0}^{b} \int_{0}^{a} \frac{d^{2} \phi_{i j}(x, y)}{d x^{2}} \phi_{i j}(x, y) d x d y & I_{2, v z i j}=\int_{0}^{b} \int_{0}^{a} \frac{d \theta_{v z}(x, y)}{d x} \frac{d^{2} \theta_{v z}(x, y)}{d x^{2}} \phi_{i j}(x, y) d x d y \\
I_{3, k l i j}=\int_{0}^{b} \int_{0}^{b} \frac{d^{2} \psi_{k l}(x, y)}{d x d y} \phi_{i j}(x, y) d x d y & I_{4, v z i j}=\int_{0}^{b} \int_{0}^{a} \frac{d \theta_{v z}(x, y)}{d y} \frac{d^{2} \theta_{v z}(x, y)}{d x d y} \phi_{i j}(x, y) d x d y
\end{array}
$$




$$
\begin{aligned}
& I_{5, i j}=\int_{0}^{b} \int_{0}^{a} \frac{d^{2} \phi_{i j}(x, y)}{d y^{2}} \phi_{i j}(x, y) d x d y \quad I_{6, v z i j}=\int_{0}^{b} \int_{0}^{a} \frac{d \theta_{v z}(x, y)}{d x} \frac{d^{2} \theta_{v z}(x, y)}{d y^{2}} \phi_{i j}(x, y) d x d y \\
& I_{7, i j}=\int_{0}^{b} \int_{0}^{a} \phi_{i j}^{2}(x, y) d x d y \quad I_{8, k l}=\int_{0}^{b} \int_{0}^{a} \frac{d^{2} \psi_{k l}(x, y)}{d y^{2}} \psi_{k l}(x, y) d x d y \\
& I_{9, v z k l}=\int_{0}^{b} \int_{0}^{a} \frac{d \theta_{v z}(x, y)}{d y} \frac{d^{2} \theta_{v z}(x, y)}{d y^{2}} \psi_{k l}(x, y) d x d y \quad I_{10, i j k l}=\int_{0}^{b} \int_{0}^{a} \frac{d^{2} \phi_{i j}(x, y)}{d x d y} \psi_{k l}(x, y) d x d y \\
& I_{11, v z k l}=\int_{0}^{b} \int_{0}^{a} \frac{d \theta_{v z}(x, y)}{d x} \frac{d^{2} \theta_{v z}(x, y)}{d x d y} \psi_{k l}(x, y) d x d y \quad I_{12, k l}=\int_{0}^{b} \int_{0}^{a} \frac{d^{2} \psi_{k l}(x, y)}{d x^{2}} \psi_{k l}(x, y) d x d y \\
& I_{13, v z k l}=\int_{0}^{b} \int_{0}^{a} \frac{d \theta_{v z}(x, y)}{d y} \frac{d^{2} \theta_{v z}(x, y)}{d x^{2}} \psi_{k l}(x, y) d x d y \quad I_{14, v z k l}=\int_{0}^{b} \int_{0}^{a} \psi_{k l}^{2}(x, y) d x d y \\
& I_{15, v z}=\int_{0}^{b} \int_{0}^{a} \frac{d^{4} \theta_{v z}(x, y)}{d x^{4}} \theta_{v z}(x, y) d x d y \quad I_{16, v z}=\int_{0}^{b} \int_{0}^{a} \frac{d^{4} \theta_{v z}(x, y)}{d x^{2} d y^{2}} \theta_{v z}(x, y) d x d y \\
& I_{17, v z}=\int_{0}^{b} \int_{0}^{a} \frac{d^{4} \theta_{v z}(x, y)}{d y^{4}} \theta_{v z}(x, y) d x d y \quad I_{18, i j v z}=\int_{0}^{b} \int_{0}^{a} \frac{d \phi_{i j}(x, y)}{d x} \frac{d^{2} \theta_{v z}(x, y)}{d x^{2}} \theta_{v z}(x, y) d x d y \\
& I_{19, v z}=\int_{0}^{b} \int_{0}^{a}\left(\frac{d \theta_{v z}(x, y)}{d x}\right)^{2} \frac{d^{2} \theta_{v z}(x, y)}{d x^{2}} \theta_{v z}(x, y) d x d y \\
& I_{20, k l v z}=\int_{0}^{b} \int_{0}^{a} \frac{d \psi_{k l}(x, y)}{d y} \frac{d^{2} \theta_{v z}(x, y)}{d y^{2}} \theta_{v z}(x, y) d x d y \\
& I_{21, v z}=\int_{0}^{b} \int_{0}^{a}\left(\frac{d \theta_{v z}(x, y)}{d y}\right)^{2} \frac{d^{2} \theta_{v z}(x, y)}{d y^{2}} \theta_{v z}(x, y) d x d y \\
& I_{22, k l v z}=\int_{0}^{b} \int_{0}^{a} \frac{d \psi_{k l}(x, y)}{d y} \frac{d^{2} \theta_{v z}(x, y)}{d x^{2}} \theta_{v z}(x, y) d x d y \\
& I_{23, v z}=\int_{0}^{b} \int_{0}^{a}\left(\frac{d \theta_{v z}(x, y)}{d y}\right)^{2} \frac{d^{2} \theta_{v z}(x, y)}{d x^{2}} \theta_{v z}(x, y) d x d y \\
& I_{24, i j v z}=\int_{0}^{b} \int_{0}^{a} \frac{d \phi_{i j}(x, y)}{d x} \frac{d^{2} \theta_{v z}(x, y)}{d y^{2}} \theta_{v z}(x, y) d x d y \\
& I_{25, v z}=\int_{0}^{b} \int_{0}^{a}\left(\frac{d \theta_{v z}(x, y)}{d x}\right)^{2} \frac{d^{2} \theta_{v z}(x, y)}{d y^{2}} \theta_{v z}(x, y) d x d y \\
& I_{26, i j v z}=\int_{0}^{b} \int_{0}^{a} \frac{d \phi_{i j}(x, y)}{d y} \frac{d^{2} \theta_{v z}(x, y)}{d x d y} \theta_{v z}(x, y) d x d y \\
& I_{27, k l v z}=\int_{0}^{b} \int_{0}^{a} \frac{d \psi_{k l}(x, y)}{d x} \frac{d^{2} \theta_{v z}(x, y)}{d x d y} \theta_{v z}(x, y) d x d y
\end{aligned}
$$




$$
\begin{aligned}
I_{28, v z}= & \int_{0}^{b} \int_{0}^{a} \frac{d \theta_{v z}(x, y)}{d x} \frac{d \theta_{v z}(x, y)}{d y} \frac{d^{2} \theta_{v z}(x, y)}{d x d y} \theta_{v z}(x, y) d x d y \\
I_{29, i j v z}= & \int_{0}^{b} \int_{0}^{a} \frac{d \theta_{v z}(x, y)}{d x} \phi_{i j}(x, y) \theta_{v z}(x, y) d x d y \\
I_{30, k l v z}= & \int_{0}^{b} \int_{0}^{a} \frac{d \theta_{v z}(x, y)}{d y} \psi_{k l}(x, y) \theta_{v z}(x, y) d x d y \\
I_{31, v z}= & \int_{0}^{b} \int_{0}^{a} \theta_{v z}^{2}(x, y) d x d y \quad I_{32, v z}=\theta_{v z}\left(x=x_{0}(t), y=b / 2\right) \\
I_{33, v z}= & \frac{d^{2} \theta_{v z}\left(x=x_{0}(t), y=b / 2\right)}{d x^{2}} \theta_{v z}\left(x=x_{0}(t), y=b / 2\right) \\
I_{34, v z}= & \theta_{v z}^{2}\left(x=x_{0}(t), y=b / 2\right) \quad I_{35, v z}=\frac{d \theta_{v z}\left(x=x_{0}(t), y=b / 2\right)}{d x} \theta_{v z}\left(x=x_{0}(t), y=b / 2\right)
\end{aligned}
$$

\section{Acknowledgment}

This paper is dedicated to Ahmad Mamandi's dearest Professor Dr. Mohammad Hossein Kargarnovin, who passed away on Monday, November 4, 2013. He was a fine, kind, decent, sincere, good tempered, and well-liked professor who will be sadly missed by all who knew him. May his soul rest in peace.

\section{References}

1. Amabili M., 2004, Nonlinear vibrations of rectangular plates with different boundary conditions: theory and experiments, Computers and Structures, 82, 2587-2605

2. Eftekhari S.A., JAfAri A.A., 2012, Vibration of an initially stressed rectangular plate due to an accelerated traveling mass, Scientia Iranica, 19, 5, 1195-1213

3. ESEN I., 2013, A new finite element for transverse vibration of rectangular thin plates under a moving mass, Finite Elements in Analysis and Design, 66, 26-35

4. FRYBA L., 1999, Vibration of Solids and Structures under Moving Loads, London: Thomas Telford Publishing

5. Gbadeyan J.A., Dada M.S., 2006, Dynamic response of a Mindlin elastic rectangular plate under a distributed moving mass, International Journal of Mechanical Science, 48, 323-340

6. Ghafoori E., Asghari M., 2010, Dynamic analysis of laminated composite plates traversed by a moving mass based on a first-order theory, Composite Structures, 92, 1865-1876

7. Ghafoori E., Kargarnovin M.H., Ghahremani A.R., 2010, Dynamic responses of rectangular plate under motion of an oscillator using a semi-analytical method, Journal of Vibration and Control, 17, 9, 1310-1324

8. Huang M.H., Thambiratnam D.P., 2001, Deflection response of plate on Winkler foundation to moving accelerated loads, Engineering Structures, 23, 1134-1141

9. LAw S.S., Bu J.Q., Zhu Z.Q., Chan S.L., 2007, Moving load identification on a simply supported orthotropic plate, International Journal of Mechanical Science, 49, 1262-1275

10. LeIssa A.W., 1969, Vibration of Plates, Washington D.C., US Government Printing Office

11. Mamandi A., Kargarnovin M.H., 2011a, Dynamic analysis of an inclined Timoshenko beam traveled by successive moving masses/forces with inclusion of geometric nonlinearities, Acta Mechanica, 218, 1, 9-29 
12. Mamandi A., Kargarnovin M.H., 2011b, Nonlinear dynamic analysis of an inclined Timoshenko beam subjected to a moving mass/force with beam's weight included, Shock and Vibration, 18, 6, $875-891$

13. Mamandi A., Kargarnovin M.H., 2013, Nonlinear dynamic analysis of an axially loaded rotating Timoshenko beam with extensional condition included subjected to general type of force moving along the beam length, Journal of Vibration and Control, 19, 16, 2448-2458

14. Mamandi A., Kargarnovin M.H., 2014, Nonlinear dynamic analysis of a Timoshenko beam resting on a viscoelastic foundation and traveled by a moving mass, Shock and Vibration, Article ID 242090, 1-10

15. Mamandi A., Kargarnovin M.H., Farsi S., 2010a, An investigation on effects of traveling mass with variable velocity on nonlinear dynamic response of an inclined Timoshenko beam with different boundary conditions, International Journal of Mechanical Sciences, 52, 1694-1708

16. Mamandi A., Kargarnovin M.H., Farsi S., 2013, Nonlinear vibration solution of an inclined Timoshenko beam under the action of a moving force with constant/non-constant velocity, Nonlinear Oscillations, 16, 3, 385-407

17. Mamandi A., Kargarnovin M.H., Younesian D., 2010b, Nonlinear dynamics of an inclined beam subjected to a moving load, Nonlinear Dynamics, 60, 277-293

18. Meirovitch L., 1997, Principles and Techniques of Vibrations, New Jersey, Printice-Hall Inc.

19. Mohebpour S.R., Malekzadeh P., Ahmadzadeh A.A., 2011, Dynamic analysis of laminated composite plates subjected to a moving oscillator by FEM, Composite Structures, 93, 1574-1583

20. NAYfeh A.H., Mook D.T., 1995, Nonlinear Oscillations, New York, Wiley-Interscience

21. Timoshenko S.P., 1959, Theory of Plates and Shells, New York, Mc Graw-Hill

22. Ugural A.C., 1999, Stresses in Plates and Shells, Singapore, McGraw-Hill

23. Vaseghi Amiri J., Nikkhoo A., Davoodi M.R., Ebrahimzadeh Hassanabadi M., 2013 , Vibration analysis of a Mindlin elastic plate under a moving mass excitation by eigenfunction expansion method, Thin-Walled Structures, 62, 53-64

24. WU J.-J., 2003, Vibration of a rectangular plate undergoing forces moving along a circular path, Finite Elements in Analysis and Design, 40, 41-60

25. Wu J.-J., 2005, Dynamic analysis of a rectangular plate under a moving line load using scale beams and scaling laws, Computers and Structures, 83, 1646-1658

26. Wu J.-J., 2007, Vibration analyses of an inclined flat plate subjected to moving loads, Journal of Sound and Vibration, 299, 373-387 